\title{
Synthesis and characterization of poly thiophene-2-carbaldehyde by hydrochloric acid as catalyst
}

\author{
Ahmad Al-Hamdan ${ }^{1, *}$ Ahmad Al-Falah ${ }^{2}$ Fawaz Al-Deri ${ }^{3}$ \\ 1. Ph.D. student in Department of Chemistry Damascus University. \\ 2.Dept. of Chemistry Damascus University and Faculty of Pharmacy Arab International. \\ ${ }^{3}$ Dept. of Chemistry Damascus University.
}

\begin{abstract}
Poly thiophene and its derivatives are important conductive polymers because of their wide applications. They were polymerized by chemical and electrochemical methods. In this paper, thiophene-2-carbaldehyde was polymerized by hydrochloric acid as a catalyst in alcohol as a solvent. The resulting polymer was characterized by (FT/IR, ${ }^{1} \mathrm{H}-\mathrm{NMR}$, EDX, and XPS) to determine the polymer structure. The resulting polymer was scanned by scanning electron microscope (SEM) for its morphological properties. We found the polymer consisting of spherical particles with a rough surface (average diameters of 127 nanometers) that clump together and form clusters
\end{abstract}

Keywords: EDX; polymers characterization; thiophene; thiophene-2-carbaldehyde; XPS.

\section{Introduction:}

Polymers are useful materials because of their ease of fabrication, flexibility, lightness of weight, and chemical inertness ( $\mathrm{Ng}$ et al., 1998). The conductive polymers were discovered in of 20th century. Which were had wide range of applications (ValderramaGarcía et al., 2016). The conductive polymer was used in sensors, bio-sensors (Mirsakiyeva, 2017), gas separation membranes (Shim and Foulger, 2012), membranes (Kasa and Gebrewold, 2017), solar cells (Duan et al., 2012), optical displays (Hernandez, 2007), light-emitting diodes (Bayramoğlu et al., 2009), rechargeable batteries (Gherras et al., 2018) and electrochromic devices (Carrasco et al., 2006). Pyrrole, furan, thiophene, and their derivatives were common monomers for synthesizing conductive polymers (Kumar et al., 2015). Thiophene derivatives have been used to synthesis polymers with wide applications such as solar cells and photoelectric cells (Raj et al., 2013).
Polythiophene gets important attention in research and industrial parts owing to its high environmental stability, better thermal stability, and mechanical characteristics. Moreover, its interesting properties like semiconducting, electronic, and optical activities along with less band gap energy (Ramesan and Suhailath, 2017). Some usually arrayed methods for the synthesis of polythiophene comprise self-assembly, template synthesis, chemical polymerization, UV-irradiation, gamma radiation, oxidative chemical polymerization, electrochemical polymerization, and so forth. Research findings have revealed that polythiophene morphology and physicochemical properties depend upon the synthesis mode (Bhattarai et al., 2019). The derivative of 2-carboxy aldehyde thiophene is polymerized by acid (RSO3H). The polymerization took place at the expense of the aldehyde group by the electrophilic addition mechanism. The polymer was reduced by hydrazine to obtain the final product (Zaman and Perepichka, 2005). 
In this research, we present the synthesis of poly(thiophene-2-carbaldehyde) (PFTh) by acid Catalysis in alcohol as a solvent, characterization by FT/IR, ${ }^{1} \mathrm{H}-\mathrm{NMR}$, XPS, EDX, SEM and AFM. And a study of its morphology.

\section{Experimental}

\subsection{Materials and Apparatus}

Thiophene-2-carbaldehyde $\quad 98.5 \%$ Sigma, Hydrochloric 35.5\% acid Sigma, methanol 98\% Sigma, UV spectrometer is Optizen model OUV322, IR spectrometer is JASCO model M4100, SEM is TESCAN model MIRA3, and ${ }^{1} \mathrm{H}-\mathrm{NMR}$ spectrometer is VARIAN model Inova 500MHz.

\subsection{Synthesis}

The thiophene-2-carbaldehyde $(50 \mathrm{mmol}$, $5.72 \mathrm{~g})$ was dissolved in the methanol $(25$ $\mathrm{ml})$, and hydrochloric acid 35.5\% (10 ml) was added. The reaction solution was left at room temperature for forty-eight hours. Firstly, the reaction solution becomes yellow than black, and in the end, form a precipitate of black color. The precipitate was filtered and washed with $\mathrm{KOH}(5 \%)$ solution, ionized water, and alcohol several times and dried at $105{ }^{\circ} \mathrm{C}$ for 48 hours. It was kept for later study.

\section{Results and discussion}

Poly(thiophene-2-carbaldehyde) was a dark greenish-black powder insoluble in common organic solvents such as alcohols, ethers, and acetonitrile. However, it was partially soluble in acetone, THF, DMF, DMSO, and formic acid. It melts at $313^{\circ} \mathrm{C}$ and decomposes.

\subsection{UV-Vis spectra}

A reaction mixture contains thiophene-2carbaldehyde (different concentrations) in alcohol and hydrochloric acid; the solutions were left at room temperature for 24 hours to finish the reaction. Then the UV-Vis spectrum was recorded on the blank of alcohol and acid. (Figure 1) shows the UVVis spectra.

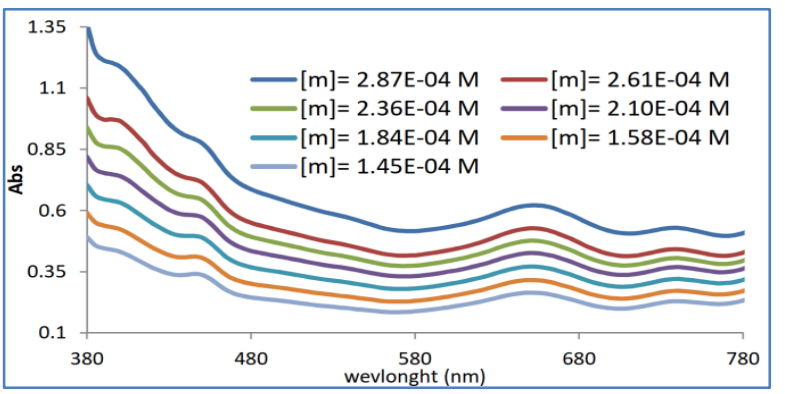

Fig. 1. UV-Vis spectra of reaction mixture solutions

The absorption spectrums (Figure 1) had three maximum absorption peaks $(443,657$, $746 \mathrm{~nm}$ ). (Figure 2) shows absorption (at maximum absorption peaks) versus concentration. The value of molecular absorption coefficient is (2755.2, 2056.2, $1728.81 / \mathrm{mol} . \mathrm{cm}$ ) respectively.

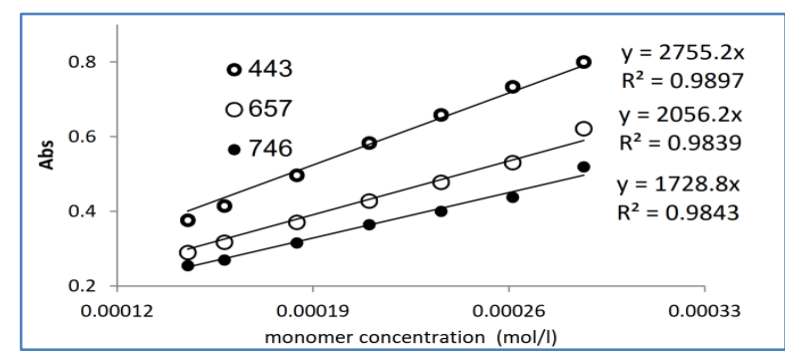

Fig. 2. Absorption (at maximum absorption peak) vs. concentration.

\subsection{FT/IR spectrophotometer:}

(Figure 3) shows FT/IR spectrum for monomer and polymer, FT/IR spectrum for monomer (Figure 3-A) shows the presence of characteristic absorption bands at $3110 \mathrm{~cm}^{-1}$ (C-H aromatic), $2825 \mathrm{~cm}^{-1}$ (C-H aliphatic), $1683 \mathrm{~cm}^{-1} \quad(\mathrm{C}=\mathrm{O}$ aldehyde $)$, the peaks between $1500 \mathrm{~cm}^{-1}$ to $1000 \mathrm{~cm}^{-1} \quad(\mathrm{C}=\mathrm{C}$ 
thiophene range and $\mathrm{C}-\mathrm{H}$ out of plane). (Figure 3-B) FT/IR spectra of polymer had the same peaks in spectra of monomer, but peak at $1683 \mathrm{~cm}^{-1} \quad(\mathrm{C}=\mathrm{O}$ aldehyde $)$ was weakened due to consumption of aldehyde by polymerization. That is means no significant change was observed between the spectrum of thiophene-2-carbaldehyde and its polymer.

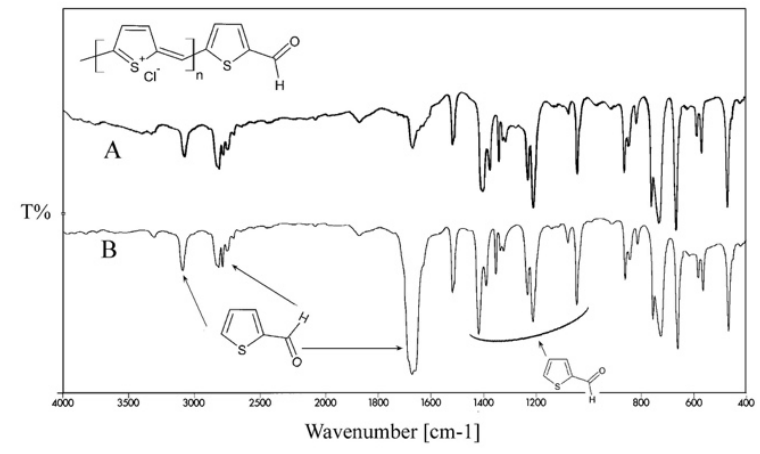

Fig. 3. FT/IR spectrum of A- polymer and Bmonomer

\section{3. ${ }^{1} \mathrm{H}-\mathrm{NMR}$ spectroscopy}

${ }^{1} \mathrm{H}$ NMR spectra of polymer data are given in (Figure 4). As seen in the $1 \mathrm{H}$ NMR spectra of polymer, the solvent's proton values were observed at (2.512 ppm, S) and others at $(3,558 \mathrm{ppm}, \mathrm{T})$ due to water insolvent.

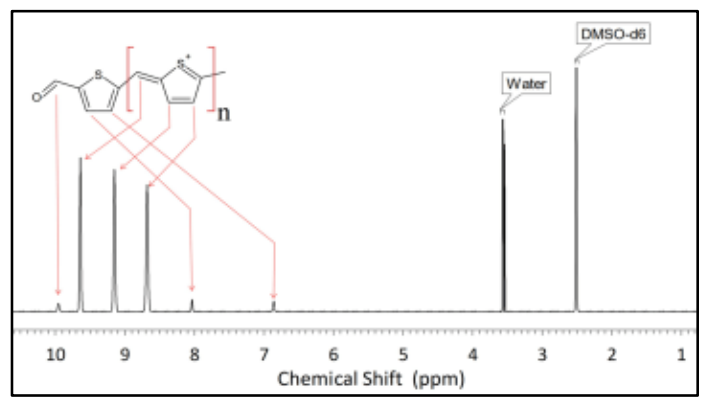

Fig. 4. ${ }^{1} \mathrm{H}$ NMR spectra of poly(thiophene-2carbaldehyde).

In addition, the proton values of the thiophene ring were observed at $(8.682 \mathrm{ppm}$, $\mathrm{S}, 1 \mathrm{H}$ and 9,148 ppm, S, 1H) and proton of methylene at $(9.643 \mathrm{ppm}, \mathrm{S}, 1 \mathrm{H})$. In $1 \mathrm{H}-$
NMR spectra of polymer found small or very small peaks due to the molecule at the tip of the polymer chain.

By the above and by the references, the following formula for polymer can be suggested.

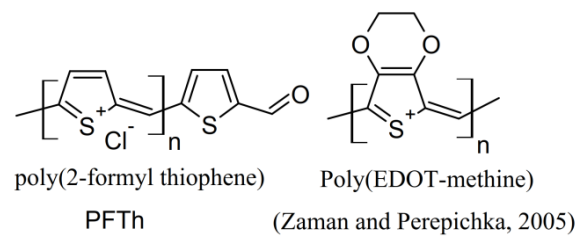

\subsection{Elemental analysis by EDX analysis}

To determine the relative composition of elements of the polymer formation, elemental analysis was performed using the EDX technique. Table 1 shows EDX results for three areas of the polymer surface. Noted that there is a slight difference due to the inaccuracy of the EDX analysis in determining the ratio of elements. The polymer contains carbon $63.0 \%$, sulfur $11.17 \%$, chlorine $12.6 \%$ and oxygen $11.2 \%$. the rate $\mathrm{S} / \mathrm{C}$ is about $21.0 \%$ (one sulfur atom for five carbon atoms). The ratio $\mathrm{S} / \mathrm{Cl}$ about one (one sulfur atom for one chlorine atom), a high percentage of oxygen due to adsorbed or trapped water in the Polymer structure.

Table 1. EDX analysis for three areas of the polymer surface.

\begin{tabular}{||lcccc||}
\hline \multirow{2}{*}{ Element } & area 1 & area 2 & area 3 & \\
& $\mathrm{Wt} \%$ & $\mathrm{Wt} \%$ & $\mathrm{Wt} \%$ & Ele/C \\
$\mathrm{C}$ & 42.46 & 40.59 & 42.43 & $100.0 \%$ \\
$\mathrm{O}$ & 9.85 & 8.23 & 11.59 & $17.7 \%$ \\
$\mathrm{~S}$ & 23.93 & 23.66 & 22.97 & $21.0 \%$ \\
$\mathrm{Cl}$ & 23.76 & 27.52 & 23.01 & $20.0 \%$ \\
Total & 100 & 100 & 100 & $100.0 \%$ \\
\hline
\end{tabular}

3.5. X-ray photoelectron spectroscopy Analysis X-ray photoelectron spectroscopy (XPS) is an excellent technique for analyzing the top 5-10 nm of a surface. (Figure 5) 
shows X-ray photoelectron spectroscopy for polymer (PFTh). The spectra had three peaks, first at $535.9 \mathrm{eV}(\mathrm{O} 1 \mathrm{~s})$, second at $287.4 \mathrm{eV}(\mathrm{C} 1 \mathrm{~s})$, and third at $157.3 \mathrm{eV}$ (S 1s).

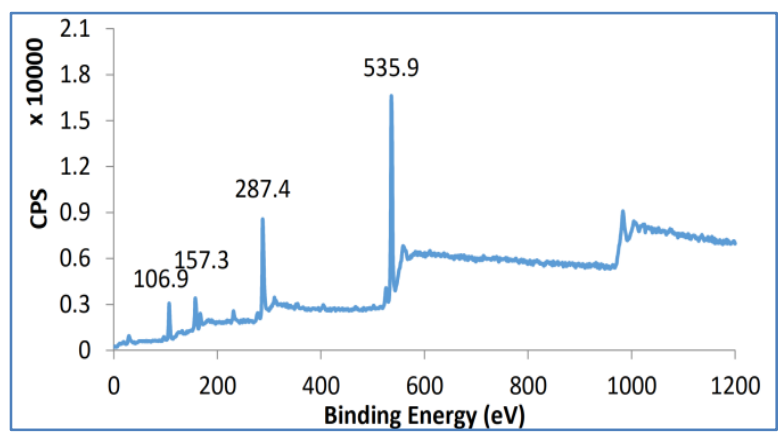

Fig. 5. X-ray photoelectron spectroscopy for (PFTh).

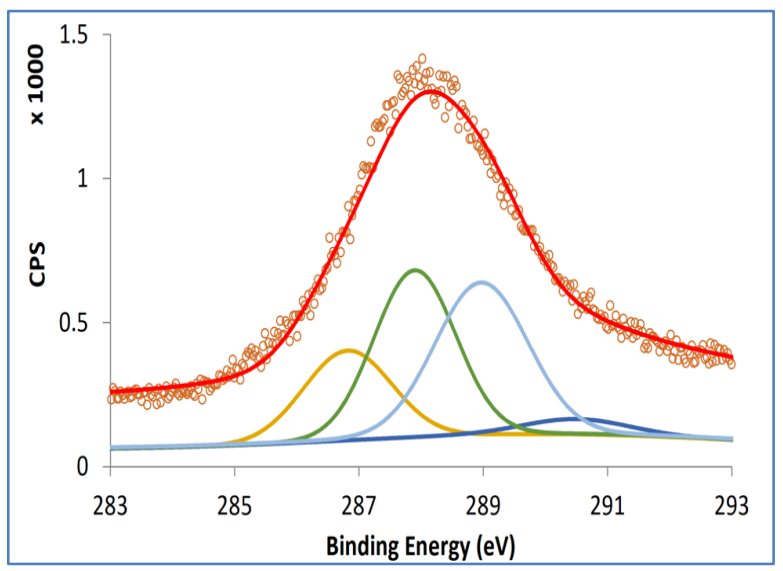

Fig. 6. (C 1s) spectra with typical peaks.

With analysis of (C 1s) spectra (Figure 6), it is possible to determine how much carbon is bound to the sulfur, hydrogen, and oxygen in the polymer structure. Table 2. shows compare between types of carbon in the polymer structure.

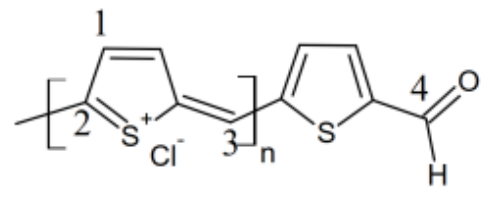

1. $\mathrm{C}-\mathrm{C}^{*}(\mathrm{H})-\mathrm{C}$ : carbon atom that bonded to carbon and hydrogen in thiophene ring at $287.9 \mathrm{eV}$ area $\%=40.69 \%$.

2. $\mathrm{C}-\mathrm{C}^{*}(\mathrm{H})-\mathrm{C}$ : carbon atom that bonded to carbon and hydrogen out of thiophene ring at $286.82 \mathrm{eV}$ area $\%=$ $19.57 \%$.

3. C-C*-S: carbon atom that bonded to carbon and sulfur in thiophene ring at $288.97 \mathrm{eV}$ area $\%=37.78 \%$.

4. $\mathrm{C}-\mathrm{C}^{*}=\mathrm{O}$ : carbon atom that bonded to carbon and oxygen in aldehyde group at $290.05 \mathrm{eV}$ area $\%=1.97 \%$.

Tebal 2. comparative between the type of carbon in polymer structure by XPS.

\begin{tabular}{||cccccc||}
\hline & Name & $\begin{array}{c}\text { BE } \\
\text { eV }\end{array}$ & $\begin{array}{c}\text { FWHM } \\
\text { eV }\end{array}$ & Area & Area\% \\
1 & $\begin{array}{c}\text { C-H } \\
\text { (ring) }\end{array}$ & 287.9 & 1.87 & 20891.57 & $40.69 \%$ \\
2 & & & & & \\
& C-H & 286.82 & 1.86 & 10047.83 & $19.57 \%$ \\
3 & C-S & 288.97 & 1.86 & 18396.12 & $37.78 \%$ \\
4 & C=O & 290.5 & 1.88 & 1010.74 & $1.97 \%$ \\
\hline
\end{tabular}

\subsection{Scanning electron microscope (SEM)}

(Figure 7) shows the photo of the scanning electron microscope (SEM) for polymer. SEM images of the resulting polymer powder (Figure 7) show the polymer being globular particles that were clump together as clusters. The maximum diameter of particles is $(587$ $\mathrm{nm})$, the minimum diameter is $(119 \mathrm{~nm})$, and the average diameter is $(121 \mathrm{~nm})$. The surface of the particles was rough and fringed with many zigzags. 


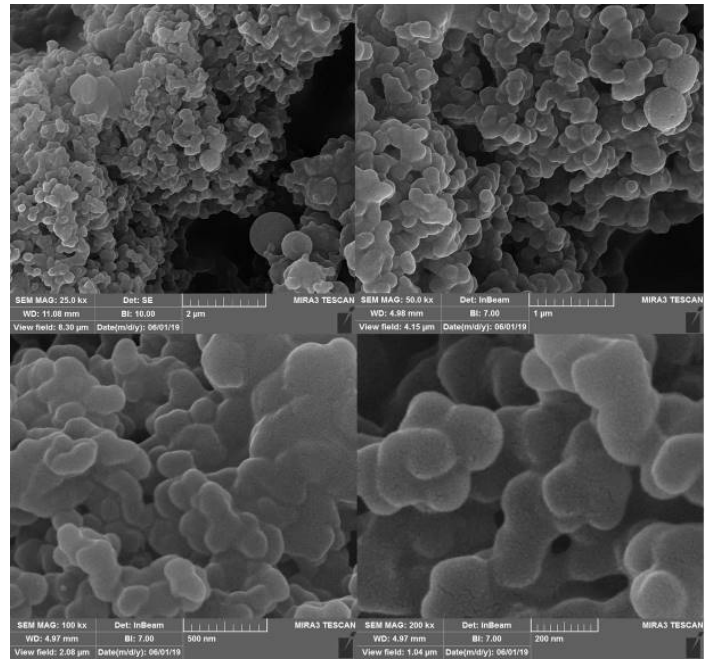

Fig. 7. shows the photo of the scanning electron microscope (SEM) for PFTh.

\subsection{Atomic force microscopy (AFM) :}

Atomic force microscopy (AFM) is used to study morphology and determine the nanoparticle size. Atomic force microscopy scans of $2 \mu \mathrm{m} \times 2 \mu \mathrm{m}$ areas of polymer film Fig 7 shows Topography, Mean diameter and 3D image polymer film. The mean diameter of polymer particles in Film where average size about $167 \mathrm{~nm}$. That differs from the size by the SEM, This may be due to the fusion of smaller particles on the surface of the film to form larger particles.

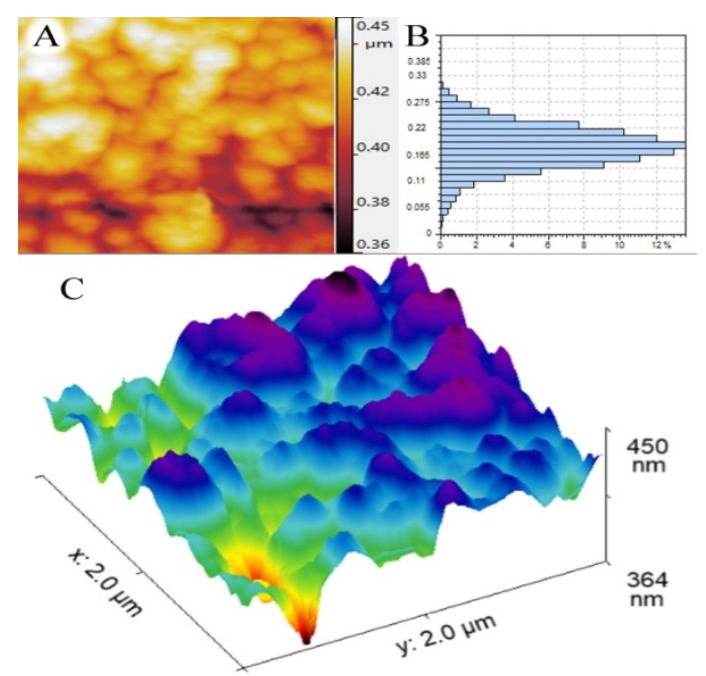

Fig. 8. A) Topography, B) Mean diameter and C) 3D image for PFTh Film.

\section{Conclusions}

Poly (thiophene-2-carbaldehyde) was synthesized by a novel, simple and easy method by adding concentrated hydrochloric acid to the monomer solution in alcohol. The polymer was characterized by FT/IR, EDX, XPS to confirm its structure. The polymer is deposited as spherical particles which have a rough surface. Its diameters were less than $600 \mathrm{~nm}$, and the average diameter was about $127 \mathrm{~nm}$ (By SEM in the synthesis conditions).

\section{References}

Bayramoğlu, G.; Karakışla, M.; Altıntaş, B.; Metin, A. U.; Saçak, M. et al. (2009). Polyaniline grafted polyacylonitrile conductive composite fibers for reversible immobilization of enzymes: Stability and catalytic properties of invertase. Process Biochemistry, $\quad$ 44(8), $\quad 880-885$. doi:10.1016/j.procbio. 2009.04.011

Bhattarai, D. P.; Awasthi, G. P.; Maharjan, B.; Lee, J.; Kim, B.-S. et al. (2019). Synthesis of polythiophene nanoparticles by surfactant-free chemical oxidative polymerization method: Characterization, in vitro biomineralization, and cytotoxicity evaluation. Journal of Industrial and Engineering Chemistry, 77(25): 243-252. doi:10.1016/j.jiec.2019.04.045.

Carrasco P. M.; Grande H. J.; Cortazar M.; Alberd. J. M I, J. Areizaga and Pomposa J. A. (2006). StructureConductivity Relationships in Chemical Polypyrroles of Low, Medium and High Conductivity, Synthetic Metals, 156 (5-6): 420-425 doi:10.1016/j.synthmet.2006.01.005. 
Duan, L.; Lu, J.; Liu, W.; Huang, P.; Wang, W. et al. (2012). Fabrication of conductive polymer-coated sulfur composite cathode materials based on layer-by-layer assembly for rechargeable lithium-sulfur batteries. Colloids and Surfaces A: Physicochemical and Engineering Aspects, 414:98-

103.doi:10.1016/j.colsurfa.2012.08.033

Gherras H.; Yahiaoui A.; Hachemaoui A.; Belfeda A.; Dehbi A. et al. (2018). synthesis and characterization of poly (2,5-diyl pyrrole-2-pyrrolyl methine) semiconductor copolymer, Journal of Semiconductors, 39(9): 102001.

Hernandez S. C. (2007). Single Polypyrrole Nanowire Ammonia Gas sensor. InterScience 19 (20): 2125-2130.

Kasa T. and Gebrewold F. (2017). Polymers and Its Application in LightEmitting Diodes: A Review Article, Advances in Physics Theories and Applications, ISSN 2225-0638 (Online), 62 (1): 28-37.

Kumar R.; Singh S. and Yadav B.C. (2015). conducting polymer: synthesis, properties, and applications, IARJSET, 2(11): 2394-2421.

Mirsakiyeva A. (2017). Electronic and optical properties of conducting polymers from quantum mechanical computations. Ph.D. thesis, KTH Royal Institute of Technology, Stockholm, Sweden.

Ng, S. C.; Chan, H. S. O.; Wong, P. M. L.; Tan, K. L., \& Tan, B. T. G. (1998). Novel heteroarylene polyazomethines: their syntheses and characterizations. Polymer, 39(20): 4963-4968. doi:10.1016/s00323861(97)10029-5.
Raj, M. R.; Anandan, S.; Solomon, R. V.; Venuvanalingam, P.; Iyer, S. S. K. et al. (2013). Conjugated polymer-based on oligobenzo[c]thiophene with low-lying HOMO energy level as a potential donor for bulk heterojunction solar cells. Journal of Photochemistry and Photobiology A: Chemistry, 262(15): 34-44. doi:10.1016/j.jphotochem.2013.04.013.

Ramesan, M. T., \& Suhailath, K. (2017). Role of nanoparticles on polymer composites. Micro and Nano Fibrillar Composites (MFCs and NFCs) from Polymer Blends, 13: 301-326. doi:10.1016/b978-008-101991-7.00013-3

Shim, G. H., \& Foulger, S. H. (2012). Coupling of crystalline colloidal arrays with intrinsically conductive polymers: Reflection-type electrochromic devices. Photonics and Nanostructures-Fundamentals and Applications, 10(4):440-446. doi:10.1016/j.photonics.2011.12.001.

Valderrama-García B.X.; Rodríguez-Alba E.; Morales-Espinoza E.G.; Chane-Ching K.M., and Rivera E. (2016). Synthesis and Characterization of Novel Polythiophenes Containing Pyrene Chromophores: Thermal, Optical, and Electrochemical Properties, Molecules, $\quad$ 21(172): 1-18. doi:10.3390/molecules21020172

Zaman M.B., and Perepichka D.F. (2005). A new simple synthesis of poly(thiophenemethine)s, The Royal Society of Chemistry, 33: 4187-4189.

Submitted: $\quad 26 / 04 / 2020$

Revised: $\quad 29 / 08 / 2020$

Accepted: $\quad 02 / 09 / 2020$

DOI: $\quad 10.48129 /$ kjs.v48i3.9624 